\title{
A Broader Perspective on the GRB-SN Connection
}

\author{
Alicia M. Soderberg \\ Division of Physics, Mathematics and Astronomy,105-24, California Institute of Technology, \\ Pasadena, CA 91125
}

\begin{abstract}
Over the last few years our understanding of local Type Ibc supernovae and their connection to long-duration gamma-ray bursts has been revolutionized. Recent discoveries have shown that the emerging picture for core-collapse explosions is one of diversity. Compiling data from our dedicated radio survey of SNe Ibc and our comprehensive HST survey of GRB-SNe together with ground-based follow-up campaigns, I review our current understanding of the GRB-SN connection. In particular, I compare local SNe Ibc with GRB-SNe based on the following criteria: (1) the distribution of optical peak magnitudes which serve as a proxy for the mass of ${ }^{56} \mathrm{Ni}$ produced in the explosion, (2) radio luminosity at early time (few days to weeks) which provides a measure of the energy coupled to on-axis relativistic ejecta, and (3) radio luminosity at late time (several years) which constrains the emission from GRB jets initially directed away from our line-of-sight. By focusing on these three points, I will describe the complex picture of stellar death that is emerging.
\end{abstract}

Keywords: Supernovae - Gamma-ray Bursts

PACS: $97.60 . \mathrm{Bw}$

\section{INTRODUCTION}

Twenty years have passed since the class of Type Ibc supernovae (SNe Ibc) was initially recognized as a distinct population of core-collapse explosions [1, 2, 3]. Their lack of homogeneity and low event rate, $(\sim 10 \%$ of locally discovered $\mathrm{SNe})$, did not motivate focused observational programs.

In 1998, however, SNe Ibc enjoyed an explosion of new-found interest thanks to the discovery of Type Ic SN $1998 \mathrm{bw}(d \approx 36 \mathrm{Mpc})$ within the BeppoSAX localization error box of gamma-ray burst, GRB $980425[4,5]$. While the $\gamma$-ray energy release, $E_{\gamma}$, of GRB 980425 was a factor of $10^{4}$ below that of GRBs, SN 1998 bw was (and still remains) the most luminous radio $\mathrm{SN}$ ever observed [6]. Two unusual features were noted from the radio data: significant energy $\left(E_{\text {radio }} \sim 10^{49} \mathrm{erg}\right)$ coupled to mildly relativistic (Lorentz factor, $\Gamma \sim 3$ ) ejecta and evidence for episodic energy injection $[6,7]$. Moreover, the bright optical emission required production of $\sim 0.5 M_{\odot}{ }^{56} \mathrm{Ni}$, comparable to that inferred for Type Ia supernovae while the broad absorption lines (indicative of photospheric velocities above $30,000 \mathrm{~km} \mathrm{~s}^{-1}$ ) implied a total kinetic energy of $3 \times 10^{52} \mathrm{erg}[8,9]$.

These observations have been interpreted under the framework of the "collapsar model" (e.g. [10]) in which a central engine (accreting black hole) plays a significant role in exploding the star. 


\section{THE GRB-SN CONNECTION: AN OVERVIEW}

In the seven years since the discovery of SN 1998bw/GRB 980425, about a dozen $\mathrm{SNe}$ Ibc have been reported in association with GRBs, all at $z \geq 0.1$ (see $[11,12]$ for recent compilations). Of these associations, three were unambiguously confirmed through spectroscopic identification of SN features (GRB 030329 [13]; GRB 031203 [14]; XRF 020903 [15]) which were observed to be unusually broad and similar to those seen in SN 1998bw. The majority of GRB-SN associations are inferred based on the emergence of a red "bump" in the afterglow light-curves approximately $20(1+z)$ days after the explosion and attributed to a thermal supernova component. These observations imply that at least some SNe Ibc are powered by a central engine.

At the same time, several broad-lined supernovae have been discovered locally and are currently estimated to represent $\sim 5 \%$ of the Type Ibc population [16]. Given their spectral similarity to SN 1998bw and GRB-SNe, it has been argued that broad-lined $\mathrm{SNe}$ Ibc can be used as signposts for GRBs, even in the absence of observed gamma-ray emission (e.g. [17]).

The question has thus become, what is the connection (if any) between the enginedriven GRB-associated SNe and local SNe Ibc? Here I present optical and radio observations for these two samples in an effort to address this question and to offer a broader perspective on the GRB-SN connection.

\section{AN OPTICAL PERSPECTIVE ON THE GRB-SN CONNECTION}

In Figure 1 I compare the peak optical magnitudes and ${ }^{56} \mathrm{Ni}$ mass estimates for GRBassociated SNe and local SNe Ibc. The figure clearly shows that ${ }^{56} \mathrm{Ni}$ mass scales with peak optical luminosity. As a result, $\mathrm{M}_{V}$ can be used as a proxy for the synthesized ${ }^{56} \mathrm{Ni}$ mass in the cases where estimates are not available. Several striking conclusions can be drawn directly from this compilation:

- SN 1998bw is not the most luminous event of either sample. In fact several local $\mathrm{SNe}$ Ibc and GRB-associated SNe are actually brighter. This emphasizes the fact that not all GRB-SNe are like SN 1998bw.

- The distributions of local and GRB-associated SNe show significant overlap. We conclude that GRB-associated SNe are not necessarily more luminous nor do they produce more ${ }^{56} \mathrm{Ni}$ than local SNe Ibc. In fact, a K-S test on the two data samples shows a $53 \%$ probability that the two have been taken from the same parent population of events. This may indicate a similar ${ }^{56} \mathrm{Ni}$ production mechanism for both samples and thus imposes significant constraints on progenitor models.

- SNe Ibc with broad optical absorption lines are not more luminous than other events. In fact, they display a range of optical luminosities comparable to the spread observed for both the local and GRB-SN samples. This emphasizes that broadoptical absorption lines cannot be used as a proxy for a large ${ }^{56} \mathrm{Ni}$ mass.

These four points illustrate the fact that optical observations cannot be used to distinguish the class of GRB-SNe from the local SNe Ibc. 


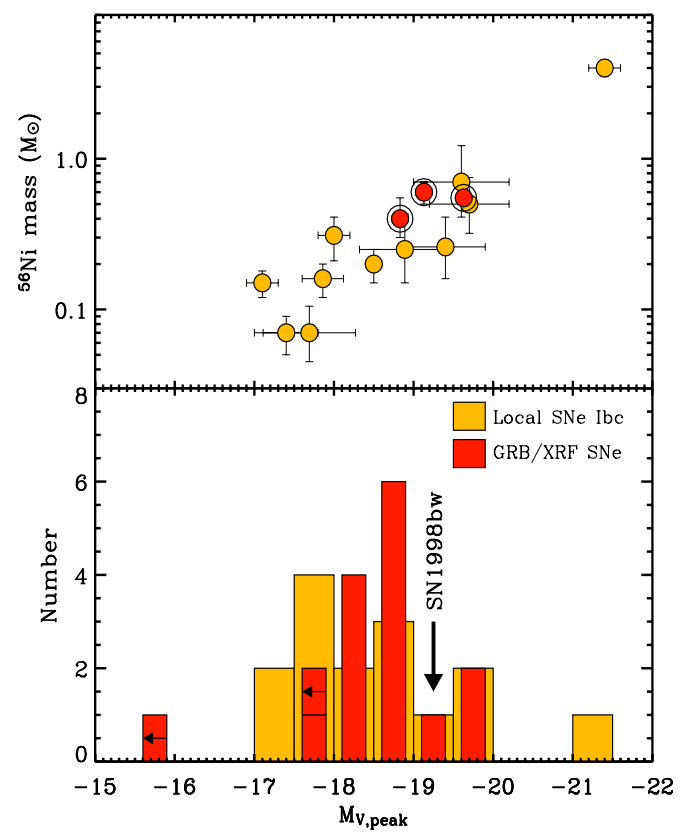

FIGURE 1. The compilation of peak optical magnitudes and ${ }^{56} \mathrm{Ni}$ mass estimates for GRB-SNe and local SNe Ibc from Soderberg et al. (2006a) has been extended to included SN 20031w/GRB 031203 (Mazzali et al., in prep) and local SNe 2003jd [17] and 2005bf [18]. The distributions for GRB-SNe and local SNe Ibc show significant overlap. A K-S test shows a 53\% probability that the two samples are drawn from the same parent population of $\mathrm{SNe}$.

\section{A RADIO PERSPECTIVE OF THE GRB-SN CONNECTION}

Radio observations offer a better way to distinguish between GRB-SNe and local $\mathrm{SNe}$ Ibc since they provide the best calorimetry of the explosion. Radio emission from $\mathrm{SNe}$ Ibc is produced by the dynamical interaction of the fastest ejecta with the circumstellar medium [19], in much the same way that GRB afterglows are produced. As the ejecta sweep up and shock the surrounding medium they produce synchrotron emission with a spectral peak near the radio band on a timescale of days to weeks. The emission is brightest for $\mathrm{SNe}$ with copious energy coupled to (mildly) relativistic ejecta, as in the case of SN 1998bw. Radio observations are therefore unique in that they provide a measure of the speed and energy of the fastest ejecta produced in the explosion.

Motivated thus, since 1999 we have been monitoring local SNe Ibc with the Very Large Array on a timescale of days to years after the explosion. Early observations are used to probe on-axis ejecta components while late-time data constrain components that were initially directed away from our line-of-sight. This six year effort has resulted in several key advances in our understanding of the GRB-SN connection. 


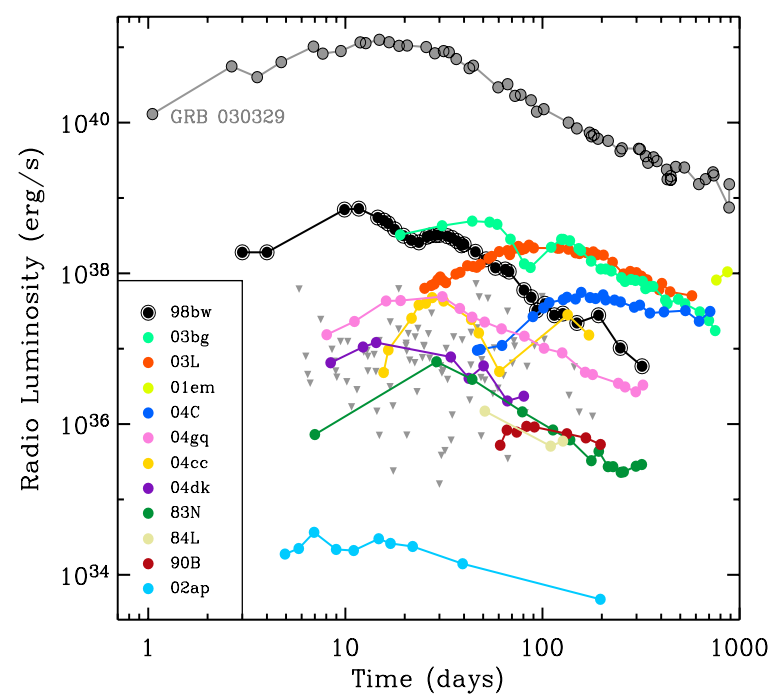

FIGURE 2. A compilation of SNe Ibc radio observations resulting mainly from our dedicated VLA survey (Soderberg et al. in prep). Upper limits are shown as inverted grey triangles. All detections have been studied as part of our VLA program with the exception of SNe 1990B, 1984L, 1983N, 2001em and $1998 \mathrm{bw}$ which were taken from the literature [20, 21, 22, 23, 6]. For comparison we show the radio lightcurve of GRB 030329 which had a radio luminosity typical of long duration GRBs [24]. These radio data show that there is a clear distinction between local SNe Ibc and GRB-SN explosions.

\section{Early-Time Observations}

First, through our extensive sample of 146 local SNe Ibc, we now know that only $10 \%$ have detectable radio emission on a timescale of a few days to years (Figures 2 and 3 ). These events typically peak in the radio band several weeks after the explosion with average luminosities a factor of $10^{2}$ times fainter than SN 1998bw on a comparable timescale. We compare the optical and radio properties for this sample of SNe Ibc and find no strong correlations. In particular, broad-lined $\mathrm{SNe}$ Ibc are not more radio luminous than the rest of the sample and can be significantly fainter (e.g. SN 2002ap, [25]; SN 2003jd, [26]). We conclude that radio bright SN 1998bw-like events are rare: less than $2 \%$ of the local population ([27]; Soderberg et al., in prep).

Next, we find a clear distinction between local SN Ibc explosions and cosmological GRBs. As clearly shown in Figure 2, GRB-SN explosions are a factor of about $10^{4}$ times more radio luminous than typical SNe Ibc. This is attributed to the fact that GRB$\mathrm{SN}$ explosions couple the bulk of their energy to highly relativistic ejecta while $\mathrm{SNe} \mathrm{Ibc}$ couple a relatively tiny fraction. These results strongly suggest that if central engines power the majority of local SNe Ibc, the engines must be weaker than those of GRB-SN explosions. 


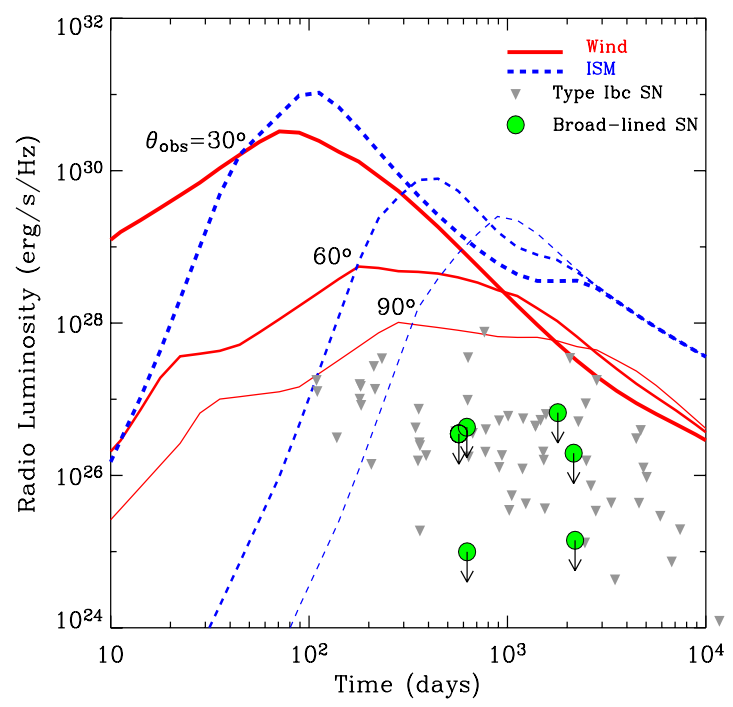

FIGURE 3. A compilation of late-time radio data for 68 local SNe Ibc as published in Soderberg et al. (2006b). Upper limits are shown as inverted grey triangles and we emphasize the limits for broad-lined $\mathrm{SNe}$ with green circles/arrows. For comparison we show the predicted radio light-curves for a typical GRB afterglow $\left(E=10^{51} \mathrm{erg}, n=A_{*}=1, \theta_{\text {jet }}=5^{\circ}\right)$ observed 30,60 and 90 degrees away from the collimation axis expanding into a constant density medium (dashed blue lines) and a stellar wind environment (solid red lines). Nearly all of the upper limits are fainter than the predicted emission from a GRB, even viewed $90^{\circ}$ off-axis. This holds in particular for the broad-lined SNe where we rule out the scenario that every broad-lined SN Ibc harbors a GRB.

\section{Late-Time Observations}

Finally, we use our late-time data obtained between 1 to 30 years after the explosion to search for evidence of relativistic GRB jets that were initially directed away from our line-of-sight. As the jets sweep up material and decelerate they spread sideways, eventually intersecting our viewing angle [28, 29]. At this point the GRB afterglow emission becomes visible and is most easily detected in the radio band. In Figure 3 we show that of the 68 events in our late-time sample, none show evidence for strong radio emission that can be attributed to an off-axis GRB jet.

We [26] compare these data to the radio luminosities of cosmological GRBs to limit the fraction of SNe Ibc hosting GRB jets to $\leq 10 \%$ (90\% confidence). This holds in particular for the broad-lined events: we rule out a scenario in which every broadlined Ibc hosts a GRB (84\% confidence). This result, taken together with the early-time radio data, reiterates that broad optical absorption lines do not imply the presence of relativistic ejecta. 


\section{CONCLUSIONS}

We compare the optical and radio properties for local SNe Ibc and GRB-SNe. We show that the optical luminosities for GRB-SNe and local SNe Ibc are comparable and therefore cannot be used to distinguish between the two samples. From our comprehensive radio survey of local $\mathrm{SNe}$, however, we are able to show a clear distinction between GRB-SNe and SNe Ibc: the radio luminosities of local events are typically $10^{4}$ times fainter than those of typical GRB-SNe and $10^{2}$ times fainter than SN 1998bw. We conclude that GRB-SN explosions couple the bulk of their energy to highly relativistic ejecta while local SNe couple a relatively tiny fraction. Finally, we use our late-time radio observations to constrain the fraction of SNe Ibc harboring off-axis GRBs to less than $10 \%$. This holds in particular for the local broad-lined SNe Ibc which have been argued (based on their spectral similarity to GRB-SNe) to be associated with off-axis GRBs. In conclusion we find that while most GRB explosions have a supernova component, only a small fraction of $\mathrm{SNe} \mathrm{Ibc}$ are capable of producing the copious relativistic ejecta characteristic of gamma-ray bursts.

\section{REFERENCES}

1. J. H. Elias, K. Matthews, G. Neugebauer, and S. E. Persson, ApJ, 296, 379 (1985).

2. J. C. Wheeler, and R. Levreault, ApJ, 294, L17 (1985).

3. A. Fillipenko, $A A R \& A, \mathbf{3 5}, 309$ (1997).

4. T. J. Galama et al., Nature, 395, 670 (1988).

5. E. Pian et al., ApJ, 536, 778 (2000).

6. S. R. Kulkarni et al., Nature, 395, 663 (1998).

7. Z.-Y. Li and R. A. Chevalier, ApJ, 526, 716 (1999).

8. K. Iwamoto et al., Nature, 395, 672 (1998).

9. S. E. Woosley et al., ApJ, 516, 788 (1999).

10. A. I. MacFadyen et al., ApJ, 550, 410 (2001).

11. A. Zeh, S. Klose, and D. H. Hartmann, ApJ, 609, 952 (2004).

12. A. M. Soderberg et al., ApJ, 636, 391 (2006a).

13. T. Matheson et al., ApJ, 599, 394 (2003).

14. D. Malesani et al., ApJL, 609, L5 (2004).

15. A. M. Soderberg et al., ApJ, 627, 877 (2005).

16. P. Podsiadlowski, P. A. Mazzali, K. Nomoto, D. Lazzati, and E. Cappellaro, ApJL, 607, L17 (2004).

17. P. A. Mazzali et al., Science, 308, 1284 (2005).

18. T. Nominaga et al., ApJL, 633, L97 (2005).

19. R. A. Chevalier, ApJ, 499, 810 (1998).

20. S. D. van Dyk, R. A. Sramek, K. W. Weiler, and N. Panagia, ApJ, 409, 162 (1993).

21. N. Panagia, R. A. Sramek, and K. W. Weiler, ApJL, 300, L55 (1986).

22. R. A. Sramek, N. Panagia, and K. W. Weiler, ApJL, 285, L59 (1984).

23. C. J. Stockdale et al., IAU Circulars, 8472, 2 (2005).

24. E. Berger et al., Nature, 426, 154 (2003).

25. E. Berger, S. R. Kulkarni, and R. A. Chevalier, ApJL, 577, L5 (2002).

26. A. M. Soderberg, E. Nakar, E. Berger, and S. R. Kulkarni, ApJ, in press (2006b), astro-ph/0507147.

27. E. Berger, S. R. Kulkarni, D. A. Frail, and A. M. Soderberg, ApJ, 599, 408 (2003).

28. B. Paczynski, ACTA Astronomica, 51, 1 (2001).

29. E. Waxman, ApJ, 602, 886 (2004). 